\title{
The distribution of generalized zeros of oscillatory solutions for second-order nonlinear neutral delay difference equations
}

\author{
Limei Feng ${ }^{1}$ and Zhenlai Han ${ }^{1 *}$
}

"Correspondence:

hanzhenlai@163.com

1School of Mathematical Sciences,

University of Jinan, Jinan, P.R. China

\section{Springer}

\begin{abstract}
In this paper, the distributions of generalized zeros of oscillatory solutions for second-order nonlinear neutral delay difference equations are studied. By means of inequality techniques, specific function sequences and non-increasing solutions for corresponding first-order difference inequality, some new estimates for the distribution of the zeros of oscillatory solutions are presented, which extend and improve some well-known results.
\end{abstract}

MSC: 35B05; 65Q10; 60E05

Keywords: Oscillation; Neutral difference equation; Distribution of zeros

\section{Introduction}

In recent years, the study of oscillation of differential equations has become more and more perfect, including various sufficient conditions, necessary conditions, the existence of non-oscillatory solutions, and even the zeros distribution of oscillatory solutions.

In 2017, Li et al. [1] studied the distribution of zeros of oscillatory solutions for secondorder nonlinear neutral delay differential equation

$$
\left(a(t) z^{\prime}(t)\right)^{\prime}+q(t) f(x(t-\sigma))=0, \quad t \geq t_{0},
$$

and obtained a sufficient condition for oscillation of differential equation.

However, most of references about oscillation of difference equations are concerned with sufficient or necessary conditions for oscillation; see [2-8]. We will also naturally ask some questions of difference equations: Are there any bounds for the distance between adjacent generalized zeros of oscillatory solutions when equations show oscillation? And how do we estimate these bounds? Therefore, we obtain the oscillation criteria of difference equations by studying the distribution of zeros.

The distribution of generalized zeros of oscillation solutions for first-order dynamic equations and second-order non-neutral dynamic equations on time scale can be found in [9-11]. However, most oscillatory results for second-order neutral dynamic equations are sufficient conditions for oscillation; see [12-19]. To the best of our knowledge, there is no paper on the generalized zero distribution of oscillation solutions for second-order neutral dynamic equations on time scale.

(c) The Author(s) 2019. This article is distributed under the terms of the Creative Commons Attribution 4.0 International License (http://creativecommons.org/licenses/by/4.0/), which permits unrestricted use, distribution, and reproduction in any medium, provided you give appropriate credit to the original author(s) and the source, provide a link to the Creative Commons license, and indicate if changes were made. 
Motivated by the above papers, we consider the second-order neutral difference equation of the following form:

$$
\Delta(a(t) \Delta z(t))+q(t) f(x(t-\sigma))=0, \quad t \in\left[t_{0}, \infty\right)_{\mathbb{Z}},
$$

where $\Delta$ denotes the forward difference operator $\Delta x(t)=x(t+1)-x(t), z(t)=x(t)+$ $p(t) x(t-\tau), \mathbb{Z}$ represents the set of all integers and

$$
\sum_{s=t_{0}}^{\infty} \frac{1}{a(s)}=\infty
$$

Throughout this paper, we assume that the following hypotheses are satisfied:

$\left(H_{1}\right) a(t), q(t), p(t) \in(0, \infty)$, where $t \in\left[t_{0}, \infty\right)_{\mathbb{Z}}$.

$\left(H_{2}\right) \tau, \sigma \in \mathbb{R}^{+}$, where $\mathbb{R}^{+}$represents the set of all positive real numbers, and $\sigma>\tau$.

$\left(H_{3}\right)$ There exists a positive constant $k$ such that $\frac{f(u)}{u} \geq k$ for all $u \neq 0$.

$\left(H_{4}\right)$ There exists a function $H(t)$ which satisfies $H(t) \geq \frac{p(t-\sigma) q(t)}{q(t-\tau)}$ and $\Delta H(t) \leq 0, t \geq t_{1}$ for some $t_{1} \geq t_{0}+\sigma$, where $t \in \mathbb{Z}$.

In this paper, we relate the distance between adjacent generalized zeros of an oscillation solution of (1.1) to a positivity problem of certain solution for a first-order delay difference inequality

$$
\Delta x(t)+P(t) x\left(t-r_{1}\right) \leq 0, \quad t \in\left[t_{0}, \infty\right)_{\mathbb{Z}}
$$

where $P(t) \in[0,1)$ which define by $(2.1), r_{1}$ is a constant satisfying $r_{1} \geq 2$.

\section{Preliminaries}

In order to prove our main results, we establish some fundamental results in this section.

For convenience, we define a sequence $\left\{F_{n}(t)\right\} \in[0,1)$ by

$$
\begin{aligned}
& F_{0}(t)=P(t):=\frac{k q(t)}{1+H(t+1)} \sum_{s=T_{0}}^{t-1} \frac{1}{a(s-\sigma)}, \quad t \in\left[t_{0}, \infty\right)_{\mathbb{Z}}, \\
& F_{n}(t)=F_{n-1}(t) \sum_{s=t-r}^{t-1} F_{n-1}(s) \prod_{\zeta=s-r}^{t} \frac{1}{1-F_{n-1}(\zeta)}, \quad t \in\left[t_{0}+2 n r, \infty\right)_{\mathbb{Z}}, n=1,2, \ldots,
\end{aligned}
$$

where $T_{0}$ satisfies $x(t)>0, t \geq T_{0}$ when $x(t)$ is eventually positive solution.

If $t_{n}$ is a generalized zero of solution of (1.1), then it satisfies $x\left(\left[t_{n}\right]\right) \cdot x\left(\left[t_{n}\right]+1\right) \leq 0$. Let $d_{s}(x)$ be the least upper bound of the distance between adjacent generalized zeros of a solution $x(t)$ of Eq. (1.1) on $[s, \infty)$.

Lemma 2.1 Assume that $x(t)$ is an eventually positive solution of (1.1), and $\left(H_{1}\right) \sim\left(H_{3}\right)$ hold. Then $z(t)$ satisfies $z(t)>0, \Delta z(t)>0, \Delta(a(t) \Delta z(t))<0$.

Proof If $x(t)$ is an eventually positive solution of Eq. (1.1), then there exists a $t_{1}>t_{0}$ such that $x(t)>0, x(t-\tau)>0$ and $x(t-\sigma)>0$ for all $t \geq t_{1}$. Thus $z(t)=x(t)+p(t) x(t-\tau)>0$. From (1.1) and condition $\left(H_{3}\right)$, we obtain

$$
\Delta(a(t) \Delta z(t))=-q(t) \frac{f(x(t-\sigma))}{x(t-\sigma)} x(t-\sigma) \leq-k q(t) x(t-\sigma)<0, \quad t \geq t_{1},
$$


so we can conclude $a(t) \Delta z(t), t \geq t_{1}$ is decreasing. It can be seen that there exists a $t_{2}>t_{1}$ such that $\Delta z(t)>0$ or $\Delta z(t)<0$ for $t \geq t_{2}$. Now, we prove $\Delta z(t)>0, t \geq t_{2}$. If not, assume that $\Delta z(t)<0, t \geq t_{2}$, then also $a(t) \Delta z(t)<-c<0$ and summing up it from $t_{2}$ to $t-1$, we have

$$
z(t)-z\left(t_{2}\right)<-c \sum_{s=t_{2}}^{t-1} \frac{1}{a(s)} .
$$

Taking limits of both sides for the above inequality, we have $\lim _{t \rightarrow \infty} z(t)=-\infty$, which is a contradiction. The proof is completed.

In the following lemmas, let $r=\left[r_{1}\right]:=\max \left\{a \mid a \leq r_{1}, a \in \mathbb{Z}\right\}$, where $r_{1}$ is the delay argument of (1.2). And $\delta$ is a constant satisfying $|\delta| \leq r$.

Lemma 2.2 Let $n$ be a positive integer such that

$$
\sum_{s=t-r}^{t-1} F_{n}(s) d s \geq 1, \quad t \in\left[t_{0}+(2 n+1) r, \infty\right)_{\mathbb{Z}}, n=1,2, \ldots
$$

If $x(t)$ is a non-increasing function on $\left[T_{1}-\delta, T\right]_{\mathbb{Z}}$ which satisfies (1.2) on $\left[T_{1}, T\right]_{\mathbb{Z}}$, then $x(t)$ cannot be positive on $\left[T_{1}, T\right]_{\mathbb{Z}}$, where $T>T_{1}+(3 n+1) r+(n+1)-\delta, T_{1} \geq t_{0}+(2 n+1) r$.

Proof Without loss of generality, we assume that $x(t)$ is positive on $\left[T_{1}, T\right]_{\mathbb{Z}}$. Summing up (1.2) from $t-r$ to $t-1$, we have

$$
x(t)-x(t-r)+\sum_{s=t-r}^{t-1} P(s) x\left(s-r_{1}\right) \leq 0, \quad t \in\left[T_{1}+r, T\right]_{\mathbb{Z}} .
$$

Multiplying this inequality by $P(t)$ and using (1.2), we get

$$
\Delta x(t)+P(t) x(t)+P(t) \sum_{s=t-r}^{t-1} P(s) x\left(s-r_{1}\right) \leq 0, \quad t \in\left[T_{1}+r+1-\delta, T\right]_{\mathbb{Z}}
$$

so

$$
\prod_{\zeta=t_{0}}^{t} \frac{1}{1-P(\zeta)}\left(\Delta x(t)+P(t) x(t)+P(t) \sum_{s=t-r}^{t-1} P(s) x\left(s-r_{1}\right)\right) \leq 0, \quad t \in\left[T_{1}+r+1-\delta, T\right]_{\mathbb{Z}} .
$$

Using $\Delta x(t)=x(t+1)-x(t)$, we get

$$
\begin{aligned}
& \prod_{\zeta=t_{0}}^{t} \frac{1}{1-P(\zeta)} x(t+1)-\prod_{\zeta=t_{0}}^{t-1} \frac{1}{1-P(\zeta)} x(t)+\prod_{\zeta=t_{0}}^{t} \frac{1}{1-P(\zeta)} P(t) \sum_{s=t-r}^{t-1} P(s) x\left(s-r_{1}\right) \leq 0, \\
& \quad t \in\left[T_{1}+r+1-\delta, T\right]_{\mathbb{Z} .}
\end{aligned}
$$

Let $y_{1}(t):=x(t) \prod_{\zeta=t_{0}}^{t-1} \frac{1}{1-P(\zeta)}$. Then $y_{1}(t)>0$ on $\left[T_{1}, T\right]_{\mathbb{Z}}$ and

$$
\Delta y_{1}(t)=\prod_{\zeta=t_{0}}^{t} \frac{1}{1-P(\zeta)} x(t+1)-\prod_{\zeta=t_{0}}^{t-1} \frac{1}{1-P(\zeta)} x(t)
$$


Using (2.7) in (2.6), we have

$$
\Delta y_{1}(t)+P(t) \sum_{s=t-r}^{t-1} P(s)\left(x\left(s-r_{1}\right) \prod_{\zeta=t_{0}}^{t} \frac{1}{1-P(\zeta)}\right) \leq 0, \quad t \in\left[T_{1}+r+1-\delta, T\right]_{\mathbb{Z}}
$$

i.e.

$$
\begin{aligned}
& \Delta y_{1}(t)+P(t) \sum_{s=t-r}^{t-1}\left(P(s) x\left(s-r_{1}\right) \prod_{\zeta=t_{0}}^{s-r-1} \frac{1}{1-P(\zeta)} \prod_{\zeta=s-r}^{t} \frac{1}{1-P(\zeta)}\right) \\
& \leq 0, \quad t \in\left[T_{1}+r+1-\delta, T\right]_{\mathbb{Z}} .
\end{aligned}
$$

From the definition of $y_{1}(t)$ and $\Delta x(t) \leq 0, t \in\left[T_{1}+r+1-\delta, T\right]_{\mathbb{Z}}$ we obtain

$$
\begin{aligned}
\Delta y_{1}(t) & =\Delta x(t) \prod_{\zeta=t_{0}}^{t} \frac{1}{1-P(\zeta)}+x(t) \Delta\left(\prod_{\zeta=t_{0}}^{t-1} \frac{1}{1-P(\zeta)}\right) \\
& =\Delta x(t) \prod_{\zeta=t_{0}}^{t} \frac{1}{1-P(\zeta)}+x(t) \prod_{\zeta=t_{0}}^{t} \frac{1}{1-P(\zeta)}-x(t) \prod_{\zeta=t_{0}}^{t-1} \frac{1}{1-P(\zeta)} \\
& =\prod_{\zeta=t_{0}}^{t} \frac{1}{1-P(\zeta)}(\Delta x(t)+x(t)-x(t)(1-P(t))) \\
& =\prod_{\zeta=t_{0}}^{t} \frac{1}{1-P(\zeta)}(\Delta x(t)+P(t) x(t)) \\
& \leq \prod_{\zeta=t_{0}}^{t} \frac{1}{1-P(\zeta)}\left(\Delta x(t)+P(t) x\left(t-r_{1}\right)\right) .
\end{aligned}
$$

Since $\Delta x(t)+P(t) x\left(t-r_{1}\right) \leq 0$, we can conclude $\Delta y_{1}(t) \leq 0, t \in\left[T_{1}+r+1-\delta, T\right]_{\mathbb{Z}}$, and from (2.8), we have

$$
\Delta y_{1}(t)+F_{1}(t) y_{1}(t-r) \leq 0, \quad t \in\left[T_{1}+3 r+1-\delta, T\right]_{\mathbb{Z}} .
$$

Repeating the above procedure to this inequality, we get

$$
\Delta y_{1}(t)+F_{1}(t) y_{1}(t)+F_{1}(t) \sum_{s=t-r}^{t-1} F_{1}(s) y_{1}(s-r) \leq 0, \quad t \in\left[T_{1}+4 r+1-\delta, T\right]_{\mathbb{Z}} .
$$

Let $y_{2}(t):=y_{1}(t) \prod_{\zeta=t_{0}+2 r}^{t-1} \frac{1}{1-F_{1}(\zeta)}$. It follows from (2.9) that

$$
\Delta y_{2}(t)+F_{1}(t) \sum_{s=t-r}^{t-1} F_{1}(s) y_{2}(s-r) \prod_{\zeta=s-r}^{t} \frac{1}{1-F_{1}(s)} \leq 0, \quad t \in\left[T_{1}+4 r+1-\delta, T\right]_{\mathbb{Z}}
$$

where $\Delta y_{2}(t) \leq 0$ for $t \in\left[T_{1}+4 r+2-\delta, T\right]_{\mathbb{Z}}$ and hence

$$
\Delta y_{2}(t)+F_{2}(t) y_{2}(t-r) \leq 0, \quad t \in\left[T_{1}+6 r+2-\delta, T\right]_{\mathbb{Z}} .
$$


Repeating this argument $n$ times, we obtain

$$
\Delta y_{n}(t)+F_{n}(t) y_{n}(t-r) \leq 0, \quad t \in\left[T_{1}+3 n r+n-\delta, T\right]_{\mathbb{Z}}
$$

where $\Delta y_{n}(t) \leq 0$ for $t \in\left[T_{1}+(3 n-2) r+n-\delta, T\right]_{\mathbb{Z}}$. Now, summing up (2.10) from $t-r$ to $t-1 \in\left[T_{1}+(3 n+1) r+n-\delta, T\right]_{\mathbb{Z}}$, we have

$$
y_{n}(t)-y_{n}(t-r)+\sum_{s=t-r}^{t-1} F_{n}(s) y_{n}(s-r) \leq 0 \text {. }
$$

Since $y(t)$ is decreasing, we obtain

$$
y_{n}(t)+y_{n}(t-r)\left[\sum_{s=t-r}^{t-1} F_{n}(s)-1\right] \leq 0, \quad t \in\left[T_{1}+(3 n+1) r+(n+1)-\delta, T\right]_{\mathbb{Z}},
$$

which is a contradiction with hypothesis (2.4). The proof of Lemma 2.2 is complete.

Lemma 2.3 Assume that $\sum_{s=t-r}^{t-2} P(s) \geq \beta$ for $0<\beta<1$ and there exist $T_{2} \geq t_{0}+r, T \geq$ $T_{2}+(1+n) r-\delta, n=1,2, \ldots$ and a function $x(t)$ satisfying inequality $(1.2)$ on $\left[T_{2}, T\right]_{\mathbb{Z}}$ with $\Delta x(t) \leq 0$ for $t \in\left[T_{2}-\delta, T\right]_{\mathbb{Z}}$. If $x(t)$ is positive on $\left[T_{2}, T\right]_{\mathbb{Z}}$, then

$$
\frac{x(t-r)}{x(t)} \geq f_{n}(\beta)>0, \quad t \in\left[T_{2}+(1+n) r-\delta, T\right]_{\mathbb{Z}}
$$

for some integer $n \geq 0$, where $f_{n}(\beta)$ is defined by

$$
f_{0}(\beta)=1, \quad f_{1}(\beta)=\frac{1}{1-\beta}, \quad f_{n+2}(\beta)=\frac{r-\beta f_{n}(\beta)}{r-\beta f_{n}(\beta)-\beta+\frac{\beta f_{n}(\beta)}{f_{n+1}(\beta)}} .
$$

Proof Since $x(t)$ is non-increasing on $\left[T_{2}-\delta, T\right]_{\mathbb{Z}}$, we find

$$
\frac{x(t-r)}{x(t)} \geq f_{0}(\beta)=1, \quad t \in\left[T_{2}+r-\delta, T\right]_{\mathbb{Z}}
$$

Summing inequality (1.2) from $t-r+1$ to $t-1$, where $t \in\left[T_{2}+2 r-\delta, T\right]_{\mathbb{Z}}$, we obtain

$$
x(t-r) \geq x(t)+\sum_{s=t-r+1}^{t-1} P(s) x\left(s-r_{1}\right) \geq x(t)+\beta x(t-r) .
$$

Therefore

$$
\frac{x(t-r)}{x(t)} \geq \frac{1}{1-\beta}=f_{1}(\beta)>0, \quad t \in\left[T_{2}+2 r-\delta, T\right]_{\mathbb{Z}}
$$

On the other hand, dividing inequality (1.2) by $x(t)$,

$$
\frac{\Delta x(t)}{x(t)}=-P(t) \frac{x\left(t-r_{1}\right)}{x(t)},
$$


because of $\Delta x(t)<0$,

$$
\frac{x(t+1)}{x(t)} \leq 1-P(t) \frac{x(t-r)}{x(t)}
$$

Multiplying from $s-r$ to $t-r-1$ where $s \in[t-r+1, t-1]_{\mathbb{Z}}$, we find

$$
\frac{x(s-r)}{x(t-r)} \geq \prod_{u=s-r}^{t-r-1} \frac{1}{1-P(u) \frac{x(u-r)}{x(u)}}, \quad t \in\left[T_{2}+3 r-\delta, T\right]_{\mathbb{Z}}
$$

According to (2.12), this yields

$$
\frac{x(s-r)}{x(t-r)} \geq \prod_{u=s-r}^{t-r-1} \frac{1}{1-f_{0}(\beta) P(u)}, \quad t \in\left[T_{2}+3 r-\delta, T\right]_{\mathbb{Z}}
$$

We can easily obtain

$$
\begin{aligned}
& \Delta\left(-\frac{1}{f_{0}(\beta)} \prod_{u=t-r}^{s-1}\left(1-f_{0}(\beta) P(u)\right)\right) \\
& =-\frac{1}{f_{0}(\beta)} \prod_{u=t-r}^{s}\left(1-f_{0}(\beta) P(u)\right)+\frac{1}{f_{0}(\beta)} \prod_{u=t-r}^{s-1}\left(1-f_{0}(\beta) P(u)\right) \\
& =-\frac{1}{f_{0}(\beta)}\left(1-f_{0}(\beta) P(s)-1\right) \prod_{u=t-r}^{s-1}\left(1-f_{0}(\beta) P(u)\right) \\
& =P(s) \prod_{u=t-r}^{s-1}\left(1-f_{0}(\beta) P(u)\right) .
\end{aligned}
$$

Combining (2.14), (2.15) with (2.13), and because of the fact

$$
\frac{n}{\frac{1}{a_{1}}+\frac{1}{a_{2}}+\cdots+\frac{1}{a_{n}}} \leq \sqrt[n]{a_{1} a_{2} \cdots a_{n}} \leq \frac{a_{1}+a_{2}+\cdots+a_{n}}{n}, \quad a_{i}>0, i=1,2, \ldots, n,
$$

we have

$$
\begin{aligned}
x(t-r)-x(t) & \geq x(t-r) \sum_{s=t-r+1}^{t-1} P(s) \frac{x(s-r)}{x(t-r)} \\
& \geq x(t-r) \sum_{s=t-r+1}^{t-1} P(s)\left(\prod_{u=s-r}^{t-r-1} \frac{1}{1-f_{0}(\beta) P(u)}\right) \\
& \geq x(t-r) \sum_{s=t-r+1}^{t-1} P(s)\left(\prod_{u=s-r}^{s-1} \frac{1}{1-f_{0}(\beta) P(u)} \prod_{u=t-r}^{s-1} 1-f_{0}(\beta) P(u)\right) \\
& \geq x(t-r) \frac{r}{r-\beta f_{0}(\beta)} \sum_{s=t-r+1}^{t-1} P(s)\left(\prod_{u=t-r}^{s-1}\left(1-f_{0}(\beta) P(u)\right)\right) \\
& =x(t-r) \frac{r}{r-\beta f_{0}(\beta)} \sum_{s=t-r+1}^{t-1} \Delta\left(-\frac{1}{f_{0}(\beta)} \prod_{u=t-r}^{s-1}\left(1-f_{0}(\beta) P(u)\right)\right)
\end{aligned}
$$




$$
\begin{aligned}
&= x(t-r) \frac{r}{r-\beta f_{0}(\beta)}\left(-\frac{1}{f_{0}(\beta)} \prod_{u=t-r}^{t-1}\left(1-f_{0}(\beta) P(u)\right)\right. \\
&\left.+\frac{1}{f_{0}(\beta)} \prod_{u=t-r}^{t-r}\left(1-f_{0}(\beta) P(u)\right)\right) \\
& \geq x(t-r) \frac{r}{r-\beta f_{0}(\beta)} \frac{1}{f_{0}(\beta)}\left(1-f_{0}(\beta) P(t-r)\right)\left(1-\frac{r-1-\beta f_{0}(\beta)}{r-1}\right) \\
& \geq x(t-r) \frac{r}{r-\beta f_{0}(\beta)} \frac{1}{f_{0}(\beta)}\left(1-\frac{f_{0}(\beta)}{f_{1}(\beta)}\right)\left(1-\frac{r-1-\beta f_{0}(\beta)}{r-1}\right) \\
& \geq x(t-r) \frac{\beta}{r-\beta f_{0}(\beta)}\left(1-\frac{f_{0}(\beta)}{f_{1}(\beta)}\right) .
\end{aligned}
$$

Thus

$$
\frac{x(t-r)}{x(t)} \geq \frac{r-\beta f_{0}(\beta)}{r-\beta f_{0}(\beta)-\beta+\frac{\beta f_{0}(\beta)}{f_{1}(\beta)}}=f_{2}(\beta)>1, \quad t \in\left[T_{2}+3 r-\delta, T\right]_{\mathbb{Z}}
$$

Repeating this argument, it follows by induction that

$$
\frac{x(t-r)}{x(t)} \geq f_{n}(\beta)>0, \quad t \in\left[T_{2}+(n+1) r-\delta, T\right]_{\mathbb{Z}}
$$

The proof is complete.

Remark It can easily be seen that either $f_{n}(\beta)$ satisfies $\lim _{t \rightarrow \infty} f_{n}(\beta)=1$ or $f_{n}(\beta)$ is nondecreasing and $\lim _{t \rightarrow \infty} f_{n}(\beta)=\infty$ or $f_{n}(\beta) \rightarrow \infty$ after finite number of terms or $f_{n}(\beta)$ is negative.

Lemma 2.4 Assume that $\sum_{s=t-r}^{t-2} P(s) \geq \beta, t \geq t_{0}$ holds for some $0<\beta<1$ and there exists a function $x(t)$ satisfying inequality (1.2) on $\left[T_{2}, T+N r+1\right]_{\mathbb{Z}}$ for some positive integer $N$ such that $\Delta x(t) \leq 0$ on $\left[T_{2}-\delta, T_{2}+N r+1\right]_{\mathbb{Z}}$ where $T_{2} \geq t_{0}+r$. If $x(t)$ is positive on $\left[T_{2}, T_{2}+\right.$ $N r+1]_{\mathbb{Z}}$, then

$$
0<\frac{x(t-r)}{x(t)} \leq g_{m}(\beta), \quad t \in\left[T_{2}+2 r-\delta, T_{2}+(N-m) r+1\right]_{\mathbb{Z}}
$$

where $m$ is a positive integer, $N \geq m+2-\frac{\delta}{r}$, and $g_{m}(\beta)$ is defined by

$$
g_{1}(\beta)=\frac{2(1-\beta)}{\beta^{2}}, \quad g_{m+1}(\beta)=\frac{2\left(1-\beta-\frac{1}{g_{m}(\beta)}\right)}{\beta^{2}}, \quad m=1,2, \ldots
$$

Proof From $\sum_{s=t-r}^{t-1} P(s) \geq \beta, t \geq t_{0}$, we see that $\sum_{s=t}^{t+r-1} P(s) \geq \beta$ for $t \geq T_{2}$. Summing both sides of (1.2) from $t$ to $t+r-1$, we obtain

$$
x(t)-x(t+r) \geq \sum_{s=t}^{t+r-1} P(s) x\left(s-r_{1}\right), \quad t \in\left[T_{2}+r, T_{2}+(N-1) r+1\right]_{\mathbb{Z}}
$$


Since $T_{2}+r \leq t \leq s \leq t+r-1$, it follows $T_{2} \leq t-r \leq s-r \leq t-1$. Again, summing (1.2) from $s-r$ to $t$ yields

$$
x(s-r)-x(t) \geq \sum_{u=s-r}^{t-1} P(u) x\left(u-r_{1}\right) .
$$

It is clear that $x\left(u-r_{1}\right)$ is non-increasing on $[s-r, t+1]_{\mathbb{Z}} \subseteq\left[T_{2}+r-\delta, T_{2}+(N-1) r+1\right]_{\mathbb{Z}}$. Thus,

$$
\begin{aligned}
x(s-r) & \geq x(t)+\sum_{u=s-r}^{t-1} P(u) x\left(u-r_{1}\right) \\
& \geq x(t)+x(t-r) \sum_{u=s-r}^{t-1} P(u) \\
& =x(t)+x(t-r)\left[\sum_{u=s-r}^{s} P(u)-\sum_{u=t}^{s} P(u)\right] \\
& \geq x(t)+x(t-r)\left[\beta-\sum_{u=t}^{s} P(u)\right] .
\end{aligned}
$$

In view of the last inequality and (2.17), we obtain

$$
\begin{aligned}
x(t) & \geq x(t+r)+\sum_{s=t}^{t+r-1} P(s) x\left(s-r_{1}\right) \\
& \geq x(t+r)+\sum_{s=t}^{t+r-1} P(s)\left[x(t)+x(t-r)\left(\beta-\sum_{u=t}^{s} P(u)\right)\right] \\
& \geq x(t+r)+\beta x(t)+\beta^{2} x(t-r)-x(t-r) \sum_{s=t}^{t+r-1} P(s)\left(\sum_{u=t}^{s} P(u)\right),
\end{aligned}
$$

for all $t \in\left[T_{2}+2 r-\delta, T_{2}+(N-1) r+1\right]_{\mathbb{Z}}$. As is well known, we have the identity

$$
\sum_{s=t}^{t+r-1} \sum_{u=t}^{s}(P(s) P(u))=\sum_{u=t}^{t+r-1} \sum_{s=u}^{t+r-1}(P(u) P(s))=\sum_{s=t}^{t+r-1} \sum_{u=s}^{t+r-1}(P(s) P(u)) .
$$

Consequently,

$$
\sum_{s=t}^{t+r-1} \sum_{u=t}^{s}(P(s) P(u))>\frac{1}{2} \sum_{s=t}^{t+r-1} \sum_{u=t}^{t+r-1}(P(s) P(u))=\frac{1}{2}\left(\sum_{s=t}^{t+r-1} P(s)\right)^{2} \geq \frac{\beta^{2}}{2} .
$$

Substituting into (2.18),

$$
x(t) \geq x(t+r)+\beta x(t)+\frac{\beta^{2}}{2} x(t-r), \quad t \in\left[T_{2}+2 r-\delta, T_{2}+(N-1) r+1\right]_{\mathbb{Z}} .
$$

Since $x(t+r)>0$ on $\left[T_{2}+2 r-\delta, T_{2}+(N-1) r\right]_{\mathbb{Z}}$, we get

$$
\frac{x(t-r)}{x(t)}<\frac{2(1-\beta)}{\beta^{2}}=g_{1}(\beta), \quad t \in\left[T_{2}+2 r-\delta, T_{2}+(N-1) r+1\right]_{\mathbb{Z}} .
$$


On the other hand, when $t \in\left[T_{2}+2 r-\delta, T_{2}+(N-2) r+1\right]_{\mathbb{Z}}$, we have $T_{2}+2 r-\delta \leq t \leq$ $t+r \leq T_{2}+(N-1) r$. So (2.20) leads to

$$
x(t+r)>\frac{1}{g_{1}(\beta)} x(t), \quad t \in\left[T_{1}+2 r-\delta, T_{1}+(N-2) r+1\right]_{\mathbb{Z}} .
$$

Since $x(t)$ is non-increasing on $\left[T_{2}-\delta, T+N r+1\right]$, it follows that

$$
x(t+r)>\frac{1}{g_{1}(\beta)} x(t) \geq \frac{1}{g_{1}(\beta)} x(t), \quad t \in\left[T_{2}+2 r-\delta, T_{2}+(N-2) r+1\right]_{\mathbb{Z}} .
$$

From this inequality and (2.19), we obtain

$$
x(t) \geq \frac{1}{g_{1}(\beta)} x(t)+\beta x(t)+\frac{\beta^{2}}{2} x(t-r), \quad t \in\left[T_{2}+2 r-\delta, T_{2}+(N-2) r+1\right]_{\mathbb{Z}} .
$$

Rearranging,

$$
\frac{x(t-r)}{x(t)}<\frac{2\left(1-\beta-\frac{1}{g_{1}(\beta)}\right)}{\beta^{2}}=g_{2}(\beta), \quad t \in\left[T_{2}+2 r-\delta, T_{2}+(N-2) r+1\right]_{\mathbb{Z}}
$$

Repeating the above procedure, we get

$$
\frac{x(t-r)}{x(t)}<\frac{2\left(1-\beta-\frac{1}{g_{m-1}(\beta)}\right)}{\beta^{2}}=g_{m}(\beta), \quad t \in\left[T_{2}+2 r-\delta, T_{2}+(N-m) r+1\right]_{\mathbb{Z}} .
$$

The proof of Lemma 2.4 is complete.

Remark Wu and $\mathrm{Xu}[18]$ proved that $g_{m}(\beta)$ is decreasing. They found also that $g_{m+1}(\beta)>$ $\frac{1-\beta}{\beta_{2}}$ for $m=1,2, \ldots$. So when $0<\beta \leq \sqrt{2}-1$, there exists a function $g(\beta)=\frac{2\left(1-\beta-\frac{1}{g(\beta)}\right)}{\beta_{2}}$ such that $\lim _{m \rightarrow \infty} g_{m}(\beta)=g(\beta)$.

Lemma 2.5 Assume that $\sum_{s=t-r}^{t-2} P(s) \geq \beta$ holds for some $\beta>\sqrt{2}-1$ and $x(t)$ is a function satisfying inequality $(1.2)$ on $\left[T_{2}, T\right]_{\mathbb{Z}}$ with $\Delta x(t) \leq 0$ for $\left[T_{2}-\delta, T\right]_{\mathbb{Z}}, T \geq T_{2}+\left(k_{\beta}+1\right) r-\delta$, $T_{2} \geq t_{0}+r$ and $k_{\beta}$ is defined by

$$
\begin{aligned}
& k_{\beta}=\left\{\begin{array}{l}
1, \quad \beta \geq 1, \\
\min \{\alpha, \gamma\}, \quad \sqrt{2}-1<\beta<1,
\end{array}\right. \\
& \alpha=\min _{n \geq 1, m \geq 1}\left\{n+m \mid f_{n}(\beta) \geq g_{m}(\beta)\right\}, \\
& \gamma=1+\min _{n \geq 1}\left\{n \mid f_{n}(\beta)<0 \operatorname{orf}_{n+1}(\beta)=\infty\right\} .
\end{aligned}
$$

Then $x(t)$ is positive on $\left[T_{2}, T\right]_{\mathbb{Z}}$.

Proof Suppose, for the sake of contradiction, that $x(t)$ is positive on $\left[T_{2}, T\right]$. We consider two cases: 
Case $1: \beta \geq 1$. In this case $k_{\beta}=1$ and $T \geq T_{2}+2 r-\delta$. Since $\Delta x(t) \leq 0$ on $\left[T_{2}-\delta, T\right]_{\mathbb{Z}}$, we obtain

$$
x(t) \geq x\left(T_{2}+r-\delta\right), \quad t \in\left[T_{2}-\delta, T_{2}+r-\delta\right]_{\mathbb{Z}}
$$

Summing both sides of (1.2) from $T_{2}+r-\delta$ to $T_{2}+2 r-\delta-1$ and using the above inequality, we obtain

$$
\begin{aligned}
x\left(T_{2}+2 r-\delta\right) & \leq x\left(T_{2}+r-\delta\right)-\sum_{s=T_{2}+r-\delta}^{T_{2}+2 r-\delta-1} P(s) x(s-r) \\
& \leq x\left(T_{2}+r-\delta\right)-x\left(T_{2}+r-\delta\right) \sum_{s=T_{2}+r-\delta}^{T_{2}+2 r-\delta-1} P(s) \\
& =x\left(T_{2}+r-\delta\right)\left[1-\sum_{s=T_{2}+r-\delta}^{T_{2}+2 r-\delta-1} P(s)\right]<0,
\end{aligned}
$$

which is a contradiction.

Case $2: \sqrt{2}-1<\beta<1$. If $k_{\beta}=n^{*}+m^{*}$, then

$$
f_{n^{*}}(\beta) \geq g_{m^{*}}(\beta)
$$

From Lemma 2.3, it follows that

$$
\frac{x(t-r)}{x(t)} \geq f_{n^{*}}(\beta), \quad t \in\left[T_{2}+\left(n^{*}+1\right) r-\delta, T\right]_{\mathbb{Z}}
$$

On the other hand, by Lemma 2.4 we find

$$
\frac{x(t-r)}{x(t)}<g_{m^{*}}(\beta), \quad t \in\left[T_{2}+2 r-\delta, T_{2}+\left(N-m^{*} r\right)+1\right]_{\mathbb{Z}}
$$

So, when $t=T_{2}+\left(n^{*}+1\right) r-\delta$ in (2.23) and (2.24), it follows that

$$
f_{n^{*}}(\beta) \leq \frac{x\left(T_{2}+n^{*} r-\delta\right)}{x\left(T_{2}+\left(n^{*}+1\right) r-\delta\right)}<g_{m^{*}}(\beta),
$$

which contradicts (2.22). If

$$
k_{\beta}=1+\min _{n \geq 1}\left\{n \mid f_{n+1}(\beta)<0 \text { or } f_{n+1}(\beta)=\infty\right\}
$$

then Lemma 2.3 implies a contradiction and the proof is complete.

\section{Main results}

In this section, we obtain sufficient oscillation conditions for Eq. (1.1) about the distribution of generalized zeros.

Theorem 3.1 Let $\left(H_{1}\right)-\left(H_{4}\right)$ and (2.4) establish for some positive integer $n$ with $r_{1}=\sigma-\tau$. Then the equation (1.1) oscillates and $d_{\tilde{t}}(x) \leq 2 \sigma+3 n(\sigma-\tau)$, where $\tilde{t}=t_{1}+(2 n+1)(\sigma-\tau)$. 
Proof If Eq. (1.1) has a non-oscillatory solution $x(t)$, and $-x(t)$ is also the solution of Eq. (1.1), so we only consider the situation of the solution of (1.1) is eventually positive. We assume $x(t)>0$ on $\left[T_{0}, T\right]_{\mathbb{Z}}$ for some integer $T_{0} \geq \tilde{t}$ where $T>T_{0}+2 \sigma+3 n(\sigma-\tau)$. Since $z(t)=x(t)+p(t) x(t-\tau)$ for $t \in\left[T_{0}+\tau, T\right], z(t)>0$ on $\left[T_{0}+\tau, T\right]_{\mathbb{Z}}$. From inequality (2.2), we have

$$
\begin{aligned}
& \Delta(a(t) \Delta z(t)) \\
& \quad \leq-k q(t) z(t-\sigma)+k q(t) p(t-\sigma) x(t-\tau-\sigma), \quad t \in\left[T_{0}+\sigma+\tau, T\right]_{\mathbb{Z}} .
\end{aligned}
$$

Also from (2.2), we obtain $x(t-\tau-\sigma) \leq-\frac{\Delta(a(t-\tau) \Delta z(t-\tau))}{k q(t-\tau)}, t \in\left[T_{0}+\sigma+\tau, T\right]_{\mathbb{Z}}$. Then inequality (3.1) can be rewritten as

$$
\begin{aligned}
& \Delta(a(t) \Delta z(t)) \\
& \quad \leq-k q(t) z(t-\sigma)-k q(t) p(t-\sigma) \frac{\Delta(a(t-\tau) \Delta z(t-\tau))}{k q(t-\tau)} \\
& \quad=-k q(t) z(t-\sigma)-p(t-\sigma) \frac{q(t)}{q(t-\tau)} \Delta(a(t-\tau) \Delta z(t-\tau)), \quad t \in\left[T_{0}+\sigma, T\right]_{\mathbb{Z}},
\end{aligned}
$$

i.e.

$$
\begin{aligned}
& \Delta(a(t) \Delta z(t))+p(t-\sigma) \frac{q(t)}{q(t-\tau)} \Delta(a(t-\tau) \Delta z(t-\tau))+k q(t) z(t-\sigma) \\
& \quad \leq 0, \quad t \in\left[T_{0}+\sigma, T\right]_{\mathbb{Z}}
\end{aligned}
$$

We can conclude from condition $\left(H_{4}\right)$ and $\Delta(a(t) \Delta z(t))<0$,

$$
\begin{aligned}
& \Delta(a(t) \Delta z(t))+H(t) \Delta(a(t-\tau) \Delta z(t-\tau))+k q(t) z(t-\sigma) \\
& \quad \leq 0, \quad t \in\left[T_{0}+\sigma, T\right]_{\mathbb{Z}} .
\end{aligned}
$$

Let

$$
w(t)=a(t) \Delta z(t)+H(t)(a(t-\tau) \Delta z(t-\tau)), \quad t \in\left[T_{0}+\sigma+\tau, T\right]_{\mathbb{Z}}
$$

So

$$
w(t) \leq(1+H(t))(a(t-\tau) \Delta z(t-\tau)) .
$$

Differentiating both sides of (3.3), and because of (3.2), and $\Delta(a(t) \Delta z(t))<0$, we obtain

$$
\begin{aligned}
\Delta w(t) & \leq \Delta H(t)(a(t-\tau+1) \Delta z(t-\tau+1))-k q(t) z(t-\sigma) \\
& <\Delta H(t)(a(t-\tau) \Delta z(t-\tau))-k q(t) z(t-\sigma), \quad t \in\left[T_{0}+\sigma, T\right]_{\mathbb{Z}} .
\end{aligned}
$$

From (3.4), we get

$$
\Delta z(t-\sigma)=\Delta z(t+\tau-\sigma-\tau) \geq \frac{w(t+\tau-\sigma)}{a(t-\sigma)(1+H(t+\tau-\sigma))}, \quad t \in\left[T_{0}+2 \sigma, T\right]_{\mathbb{Z}}
$$


Summing up the above form from $T_{0}$ to $t-1$, we have

$$
z(t-\sigma)-z\left(T_{0}-\sigma\right) \geq \sum_{s=T_{0}}^{t-1} \frac{w(s+\tau-\sigma)}{1+H(s+\tau-\sigma)} \frac{1}{a(s-\sigma)}
$$

therefore

$$
z(t-\sigma) \geq \sum_{s=T_{0}}^{t-1} \frac{w(s+\tau-\sigma)}{1+H(s+\tau-\sigma)} \frac{1}{a(s-\sigma)}
$$

Let

$$
y(t)=\frac{w(t)}{1+H(t)}>0, \quad t \in\left[T_{0}+\sigma+\tau, T\right]_{\mathbb{Z}}
$$

Then

$$
\Delta y(t)=\frac{\Delta w(t)(1+H(t))-w(t) \Delta(1+H(t))}{(1+H(t+1))(1+H(t))}, \quad t \in\left[T_{0}+\sigma+\tau, T\right]_{\mathbb{Z}} .
$$

Adding (3.3) and (3.5) to (3.8), we have

$$
\Delta y(t)+\frac{k q(t) z(t-\sigma)}{1+H(t+1)}<0, \quad t \in\left[T_{0}+\sigma+\tau, T\right]_{\mathbb{Z}}
$$

From (3.6), (3.7) and the decreasing of $y(t)$, we get

$$
z(t-\sigma) \geq \sum_{s=T_{0}}^{t-1} \frac{1}{a(s-\sigma)} y(s+\tau-\sigma) \geq y(t+\tau-\sigma) \sum_{s=T_{0}}^{t-1} \frac{1}{a(s-\sigma)}, \quad t \in\left[T_{0}+\sigma+\tau, T\right]_{\mathbb{Z}}
$$

Substituting the above inequality into (3.9), we obtain

$$
\Delta y(t)+\frac{k q(t) y(t+\tau-\sigma)}{1+H(t+1)} \sum_{s=T_{0}}^{t-1} \frac{1}{a(s-\sigma)}<0, \quad t \in\left[T_{0}+\sigma+\tau, T\right]_{\mathbb{Z}}
$$

Set $r_{1}=\sigma-\tau, T_{1}=T_{0}+\sigma+\tau$ and $P(t)=\frac{k q(t)}{1+H(t+1)} \sum_{s=T_{0}}^{t-1} \frac{1}{a(s-\sigma)}$, we conclude

$$
\Delta y(t)+P(t) y\left(t-r_{1}\right)<0, \quad t \in\left[T_{1}, T\right]_{\mathbb{Z}}
$$

What is more, (2.1) holds and $y(t)$ is decreasing. Then we can conclude from Lemma 2.2 that $y(t)$ cannot be positive on $\left[T_{1}, T\right]_{\mathbb{Z}}$ when $r_{1}=\sigma-\tau$, where $T>T_{1}+3 n(\sigma-\tau)$. This is a contradiction with (3.7). The proof is completed.

Assume the following condition holds:

$\left(H_{5}\right) \sum_{s=t-r}^{t-1} \frac{q(s)}{1+H(s+1)} \sum_{v=T_{0}}^{s-1} \frac{1}{a(v-\sigma)} \geq \beta, t \geq t_{2}$ for some $t_{2} \geq t_{0}+2 \sigma-\tau$.

Then we can obtain some further conclusions by means of Theorem 3.1.

Corollary 3.1 Suppose conditions $\left(H_{1}\right)-\left(H_{5}\right)$ hold and a sequence $\left\{\beta_{n}\right\}$ is defined by

$$
\beta_{0}=\beta>0, \quad \beta_{n}=\beta_{0}{ }^{n+1}, \quad n=0,1,2, \ldots
$$


If there is some positive constant $n_{0} \in \mathbb{N}$ such that $1 \leq \beta<r$, then Eq. (1.1) is oscillatory and $d_{\tilde{t}} \leq 2 \sigma+3 n_{0}(\sigma-\tau)$, where $\tilde{t}=t_{1}+\left(2 n_{0}+1\right)(\sigma-\tau)$.

Proof According to condition $\left(H_{5}\right)$, we have $\sum_{s=t-r}^{t-1} F_{0}(s) \geq \beta$. In addition, from the iterative sequence $\left\{F_{n}(t)\right\}$, we get

$$
\begin{aligned}
\sum_{s=t-r}^{t-1} F_{1}(s) & =\sum_{s=t-r}^{t-1} F_{0}(s) \sum_{\nu=s-r}^{s-1} F_{0}(s) \prod_{\zeta=v-r}^{s-1} \frac{1}{1-F_{0}(\zeta)} \\
& \geq \sum_{s=t-r}^{t-1} F_{0}(s) \sum_{v=s-r}^{s-1} F_{0}(s) \prod_{\zeta=\nu-r}^{v-1} \frac{1}{1-F_{0}(\zeta)} \prod_{\zeta=v}^{s-1} \frac{1}{1-F_{0}(\zeta)} \\
& \geq \frac{r}{r-\beta} \sum_{s=t-r}^{t-1} F_{0}(s) \sum_{\nu=s-r}^{s-1} F_{0}(s) \\
& =\frac{r}{r-\beta} \beta^{2} \geq \beta^{2} .
\end{aligned}
$$

In the same way, continuing the calculation $n$ times, we obtain $\sum_{s=t-r}^{t-1} F_{n}(s) \geq \beta_{n}$ for $n=$ $2,3, \ldots$. Therefore, by mathematical induction, we have

$$
\sum_{s=t-r}^{t-1} F_{n}(s) \geq \beta_{n}, \quad \text { for all } n \in \mathbb{N}
$$

Let $n=n_{0}$. According to Theorem 3.1, the proof is completed.

Theorem 3.2 Let $\left(H_{1}\right)-\left(H_{5}\right)$ hold. Then Eq. (1.1) oscillates and $d_{t_{2}}(x) \leq 2 \sigma+k_{\beta}(\sigma-\tau)$, where $k_{\beta}$ is defined by (2.21).

Proof As usual, we assume (1.1) has a solution $x(t)>0$ on $\left[T_{0}, T\right]_{\mathbb{Z}}$ where $T>T_{0}+2 \sigma+$ $k_{\beta}(\sigma-\tau)$ and $T_{0} \geq t_{2}$. Proceed as in the proof of Theorem 3.1, when $T_{1}=T_{0}+2 \sigma$. It follows that

$$
\Delta y(t)+p(t) y(t+\tau-\sigma)<0, \quad t \in\left[T_{1}, T\right]_{\mathbb{Z}},
$$

where

$$
y(t)>0, \quad t \in\left[T_{1}-2(\sigma-\tau), T\right]_{\mathbb{Z}}
$$

Also from (3.10) we obtain

$$
\Delta y(t)<0, \quad t \in\left[T_{1}-(\sigma-\tau), T\right]_{\mathbb{Z}}
$$

Since $\left(H_{5}\right)$ holds, we conclude from Lemma 2.5 with $\delta=\sigma-\tau$ that $y(t)$ cannot be positive on $\left[T_{1}, T\right]_{\mathbb{Z}}$, where $T>T_{1}+k_{\beta}(\sigma-\tau)$. This contradiction completes the proof. 


\section{Example}

In this section, we will present an example to illustrate main results.

Example 4.1 We consider the delay difference equation

$$
\Delta(\Delta(x(t)+x(t-1)))+\frac{7}{48(t-2)} x(t-2)=0, \quad t \geq 1 .
$$

Compared with (1.1), we denote $a(t)=1$ where $c>t-1, p(t)=1, q(t)=\frac{7}{48(t-2)}, \tau=1, \sigma=3$, $r=\sigma-\tau=2, f(u)=u$ for $u \neq 0$. It is easy to verify that conditions $\left(H_{1}\right)$ and $\left(H_{2}\right)$. Since $f(u)=u$, we get $\frac{f(u)}{u}=1 .\left(H_{3}\right)$ holds. Now take $H(t) \equiv 1$ which satisfies $\left(H_{4}\right)$. According to (2.1), we obtain $F_{0}(t)=\frac{k q(t)}{1+H(t+1)} \sum_{s=T_{0}}^{t-1} \frac{1}{a(s-\sigma)}=\frac{7}{2 \times 48(t-2)} \sum_{s=2}^{t-1} 1=\frac{7}{24}, t \geq 1$ and $\frac{1}{1-F_{0}(\zeta)}=\frac{24}{17}$, so

$$
\begin{aligned}
F_{1}(t) & =F_{0}(t) \sum_{s=t-r}^{t-1} F_{0}(s) \prod_{\zeta=s-r}^{t} \frac{1}{1-F_{0}(\zeta)} \\
& =\frac{7}{24} \frac{7}{24}\left(\frac{24}{17}\right)^{5}+\frac{7}{24} \frac{7}{24}\left(\frac{24}{17}\right)^{4} \approx 0.815, \quad t \geq 6 .
\end{aligned}
$$

Therefore, $\sum_{s=t-2}^{t-1} F_{1}(s) d s \geq 1$ for all $t \geq 6$. Here, all conditions of Theorem 3.1 are satisfied with $n=1$, then we derive that (4.1) shows oscillatory and $d_{\tilde{t}}(x) \leq 2 \sigma+3 n(\sigma-\tau)=12$, where $\tilde{t}=t_{1}+(2 n+1)(\sigma-\tau)=t_{1}+6$ and $t_{1} \geq t_{0}+\sigma=4$.

\section{Conclusion}

In this paper, two theorems on the distribution of oscillation zeros for second-order nonlinear neutral delay difference equations are obtained by means of inequality techniques, specific function sequences and non-increasing solutions for corresponding first-order difference inequality. Comparing with the corresponding differential equation, it is more complex to deal with the lower bound of summation. Function $\frac{1}{a(t)} \prod_{s=1}^{t-1}(1+a(s))$ is invariant after derivation in difference equation, which is equivalent to $e^{x}$ in differential equation. That is the difficulty we address and the innovation of this paper. We study a second-order equation under the canonical form, and it is also of great significance for the study of non-canonical forms. Moreover, this paper can be extended to the dynamic equation on time scale.

\section{Acknowledgements}

The authors sincerely thank the reviewers for their valuable suggestions and useful comments that have led to the present improved version of the original manuscript.

\section{Funding}

This research is supported by the Natural Science Foundation of China (61703180, 61803176), and supported by Shandong Provincial Natural Science Foundation (ZR2017MA043).

\section{Competing interests}

The authors declare that they have no competing interests.

\section{Authors' contributions}

The authors declare that the study was realized in collaboration with the same responsibility. All authors read and approved the final manuscript. 


\section{Publisher's Note}

Springer Nature remains neutral with regard to jurisdictional claims in published maps and institutional affiliations.

Received: 2 March 2019 Accepted: 26 June 2019 Published online: 11 July 2019

\section{References}

1. Li, H., Han, Z.L., Sun, S.R.: The distribution of zeros of oscillatory solutions for second order nonlinear neutral delay differential equations. Appl. Math. Lett. 63, 14-20 (2017)

2. Erbe, L.H., Kong, Q.K., Zhang, B.G.: Oscillation Theory for Functional Differential Equations. Dekker, New York (1995)

3. Stavroulakis, I.P.: Oscillation criteria for first order delay difference equations. Mediterr. J. Math. 1, 231-240 (2014)

4. Chatzarakis, G.E., Stavroulakis, I.P.: Oscillation of first order linear delay difference equations. J. Math. Anal. Appl. 3, 1-11 (2006)

5. Chatzarakis, G.E., Stavroulakis, I.P.: Oscillation criteria of first order linear difference equations with delay arguments. Nonlinear Anal. 68, 994-1005 (2008)

6. Stavroulakis, I.P.: Oscillation criteria for delay and difference equations with non-monotone arguments. Appl. Math. Comput. 226, 661-672 (2014)

7. Chatzarakis, G.E., Leonid, S.: Oscillation criteria for difference equations with non-monotone arguments. Adv. Differ. Equ. 2017, 62 (2017)

8. Chatzarakis, G.E., Philos, C.G., Stavroulakis, I.P.: An oscillation criterion for linear difference equations with general delay argument. Port. Math. 66, 513-533 (2009)

9. Zhang, B.G., Lian, F:: The distribution of generalized zeros of solutions of delay differential equations on time scales. J. Differ. Equ. Appl. 10(8), 759-771 (2004)

10. Wu, H.: The distribution ofzZeros of solutions of advanced dynamic equations on time scales. Bull. Malays. Math. Sci. Soc. 41, 63-79 (2018)

11. Saker, S.H., Mahmoud, R.R.: Distribution of zeros of sublinear dynamic equations with a damping term on time scales. Hacet. J. Math. Stat. 45(2), 455-471 (2016)

12. Agarwal, R.P., Bohner, M., Li, T., Zhang, C.: Comparison theorems for oscillation of second-order neutral dynamic equations. Mediterr. J. Math. 11, 1115-1127 (2014)

13. Li, T., Agarwal, R.P., Bohner, M.: Some oscillation results for second-order neutral dynamic equations. Hacet. J. Math. Stat. 41,715-721 (2012)

14. Li, T., Saker, S.H.: A note on oscillation criteria for second-order neutral dynamic equations on isolated time scales. Commun. Nonlinear Sci. Numer. Simul. 19, 4185-4188 (2014)

15. Zhang, C., Agarwal, R.P., Bohner, M., Li, T.: New oscillation results for second-order neutral delay dynamic equations. Adv. Differ. Equ. 2012, 227 (2012)

16. Zhang, C., Agarwal, R.P., Bohner, M., Li, T.: Oscillation of second-order nonlinear neutral dynamic equations with noncanonical operators. Bull. Malays. Math. Sci. Soc. 38, 761-778 (2015)

17. Bohner, M., Li, T.: Oscillation of second-order $p$-Laplace dynamic equations with a nonpositive neutral coefficient. Appl. Math. Lett. 37, 72-76 (2014)

18. Wu, H.W., Xu, Y.T.: The distribution of zeros of solutions of neutral differential equations. Appl. Math. Comput. 156, 665-677 (2004)

19. Baker, A.F., El-Morshedy, H.A.: The distribution of zeros of all solutions of first order neutral differential equations. Appl. Math. Comput. 259, 777-789 (2015)

\section{Submit your manuscript to a SpringerOpen ${ }^{\circ}$ journal and benefit from:}

- Convenient online submission

- Rigorous peer review

- Open access: articles freely available online

- High visibility within the field

- Retaining the copyright to your article

Submit your next manuscript at $\gg$ springeropen.com 